\title{
Contact-free measurement and numerical and analytical evaluation of the strain distribution in a wood-FRP lap-joint
}

\author{
Johan Vessby $\cdot$ Erik Serrano $\cdot$ Bertil Enquist
}

Received: 8 May 2009/ Accepted: 26 November 2009

(C) RILEM 2009

\begin{abstract}
Wood specimens to each of which a laminate of carbon fibre reinforcement polymers (FRP) was glued (creating a lap joint in each case) were loaded to failure. A total of 15 specimens of three types differing in the glued length (anchorage length) of the FRP laminate $(50,150$ and $250 \mathrm{~mm}$ respectively) were tested, their strength, stiffness and strain distribution being evaluated. Synchronized digital cameras (charge-coupled devices) used in testing enabled strain fields on surfaces they were directed at during the loading procedure to be measured. These results were also evaluated both analytically on the basis of generalized Volkersen theory and numerically by use of the finite element method. The lap joints showed a high level of stiffness as compared with mechanical joints. A high degree of accuracy in the evaluation of stiffness was achieved through the use of the contact-free evaluation system. The load-bearing capacity of joints of this type was found to be dependent upon the
\end{abstract}

J. Vessby

Tyréns, Storgatan 40, 35231 Växjö, Sweden

J. Vessby $(\bowtie) \cdot$ E. Serrano $\cdot$ B. Enquist

Växjö University, Lückligs Plats 1, 35195 Växjö, Sweden

e-mail: johan.vessby@vxu.se

E. Serrano

e-mail: erik.serrano@vxu.se

B. Enquist

e-mail: bertil.enquist@vxu.se anchorage length in a non-linear fashion. The experimental, analytical and numerical results were shown to be in close agreement with respect to the strength and the strain distribution obtained.

Keywords FRP · Lap-joint · Volkersen · Wood

\section{Background}

In the building sector, enhancement of the loadcarrying capacity of new or already existing structures without any change in their geometry needing to be made may be sought. This may be based on changed conditions, such as an increase in loading due to rebuilding or redesigning of the overall structure. Points in close proximity to connections between different building elements or around holes or notches in beams, for example, can be in particular need of reinforcement. What is aimed at may be greater stiffness, enhanced load-bearing capacity, or both. One method of increasing the stiffness and strength of a structure is by use of Fibre Reinforced Polymers (FRP). These show a very high level of strength and stiffness as compared with many other materials.

The aim of the present study was to investigate experimentally under well-defined conditions and evaluate analytically and numerically the properties of the interaction between FRP-laminates (referred to hereafter as FRPs) and timber to which they are glued to form single-overlap joints (these are also termed 
lap-joints). Other researchers, such as Gustafsson and Enquist [1], Johansson et al. [2] and Kliger et al. [3] have reported both experimentally and numerically based findings concerning properties of the FRPtimber interaction. In these studies no use was made of a contact-free evaluation system to examine in detail the behaviour of the joint, a matter the very large amount of data obtained by use of such a system makes possible. Guan et al. [4] studied a timber beam reinforced with glass fibre pretensioned to different forces. They compared the load-deflection relations between a three-dimensional finite element model and experimental results and obtained good agreement.

One can note that experiments have also been performed with the aim of developing design equations for glued-in rods, an application that shows strong similarities to FRP overlap joints, see Steiger et al. $[5,6]$. In the present study no design parameters are proposed. The objective is rather to investigate the usefulness of various methods for analyzing FRP lap joints. One of these is the contact-free evaluation system just referred to. Two others are the use of analytical models based on generalized Volkersen theory (see [7, 8]), and analysis by use of the finite element method.

\section{Materials and methods}

\subsection{Test specimens}

Wooden test specimens of Norway Spruce $350 \mathrm{~mm}$ in length were sawn from $5 \mathrm{~m}$ long boards $70 \times$ $145 \mathrm{~mm}^{2}$ in cross-section. Prior to any further handling, the wood specimens were stored in a standard climate at $20^{\circ} \mathrm{C}$ and a relative humidity of $65 \%$ until moisture equilibrium was achieved. After completion of the experiments, conducted thereafter, the moisture content of the wood specimens was verified by oven drying and then weighting them. Just prior to the experiments, the surface on the side on which the FRP was to be attached by glue was planed.

The FRPs were $1.4 \mathrm{~mm}$ thick their stiffness and strength, as stated by the manufacturer, were 150 and $2000 \mathrm{MPa}$ respectively. All carbon fibres where oriented in the same direction. The FRPs were glued to the wood by use of a 2-component epoxy adhesive (Resin 220) using an amount of adhesive producing a bond line thickness of $1.3 \mathrm{~mm}$. The glue was applied

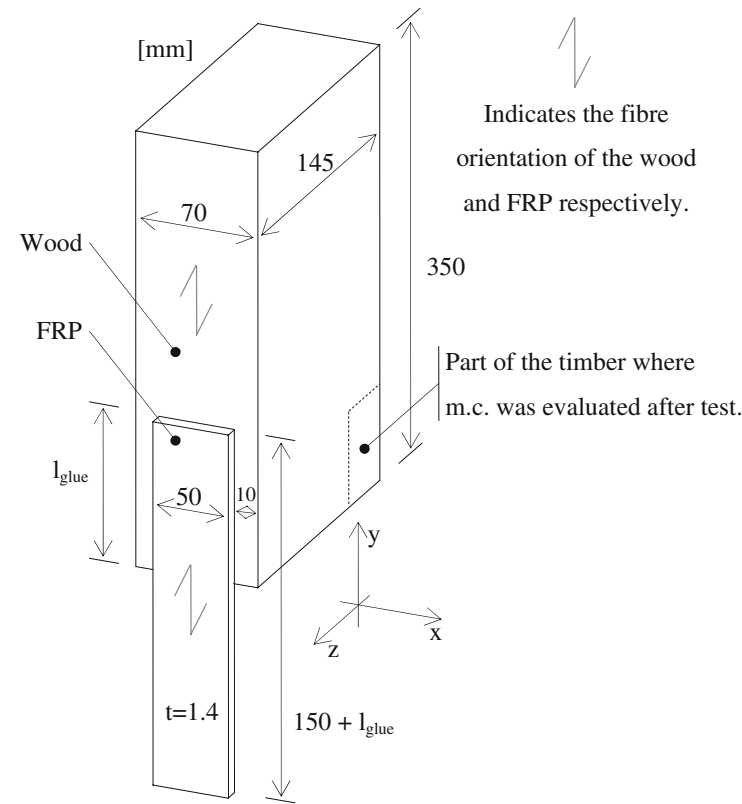

Fig. 1 The test specimens, $70 \times 145 \mathrm{~mm}^{2}$ in cross section, were sawn to $350 \mathrm{~mm}$ in length. The FRP was glued to them using three different anchorage lengths $\left(l_{\text {glue }}\right): 50,150$ and $250 \mathrm{~mm}$

by means of a spatula. Each FRP was centrically placed on one edge of the wood specimen in question, such that there was $10 \mathrm{~mm}$ clearance to the edge of the specimen, see Fig. 1. No clamping was used while attaching the two pieces to each other.

The specimens were of three different types, A, B and $\mathrm{C}$, differing in the length of the overlap, $l_{\text {glue }}$, such that the specimens in the groups had a glued overlap of 50, 150 and $250 \mathrm{~mm}$, respectively. After the FRP had been glued to the wood, the specimens were once again stored in the $20^{\circ} \mathrm{C} / 65 \%$ standard climate. For each of the three groups, five test specimens were manufactured, enabling 15 tests to be performed. The part of the FRP sticking out from the piece of wood to which it was attached was $150 \mathrm{~mm}$ in length in each case. This part of the FRP was used for applying loads by means of the hydraulic grip of the testing machine.

\subsection{Experimental setup}

The experimental setup, cf. Fig. 2, aimed at transmitting shear forces within the plane between the FRP and the timber. The specimen was fixed on its upper end-grain surface by means of a short steel 


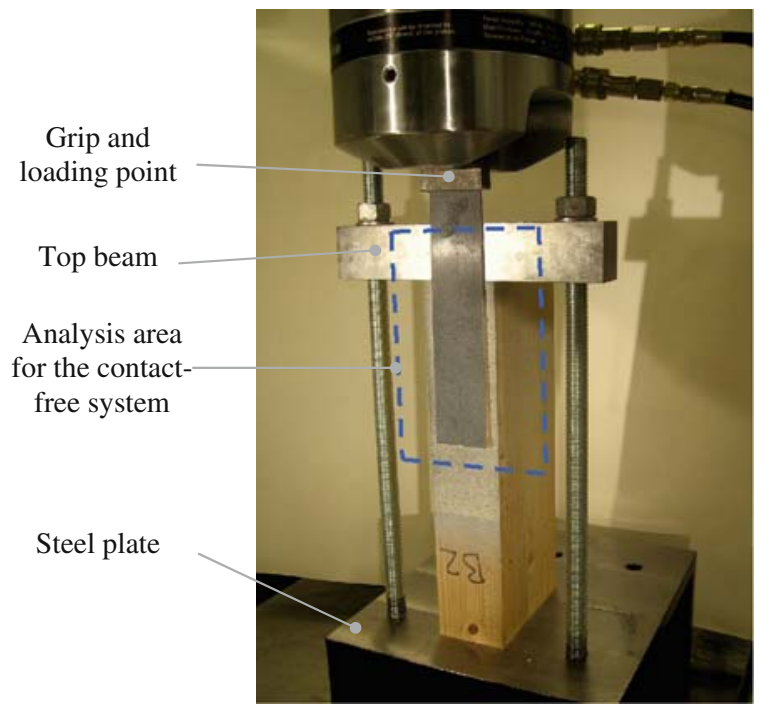

Fig. 2 Experimental setup. Testing machine. The area evaluated by means of the contact-free measurement system is indicated

beam having dimensions of $50 \times 50 \mathrm{~mm}^{2}$. The beam was held in place and in contact with the specimen by use of tie-down threaded rods of the M20 type, together with a washer and a nut. The tie-down rods were anchored to a solid steel structure (a part of the testing machine). The load was applied at the free end of the FRP by displacement-controlled movement, the displacement increasing successively at $0.5 \mathrm{~mm} /$ min. The loading rate was adjusted so as to lead to failure of the specimen within 1-3 min. The load cell employed had a capacity of $100 \mathrm{kN}$ and a linear tolerance interval of $\pm 0.1 \%$. An overview of the setup is shown in Fig. 3.

\subsection{Collection of experimental data}

Along with registering the force applied by the testing machine, deformation data was collected using two different methods: measuring the vertical displacement of the piston and utilizing a contact-free measurement system (Aramis ${ }^{\mathrm{TM}}$ ). An obvious difference between the two deformation measurement methods is that measurements of piston displacement are affected by the flexibility of the setup as a whole and of the test specimen itself, whereas using the contact-free system displacements between any two points within the field of measurements can be determined. This latter technique enables the entire field of in-plane strains to be measured continuously in the course of an experiment.

The contact-free measurement technique is based on the use of two cameras which are placed in front of the test object at angles and distances determined by the size of the object and by the lenses involved. With the two cameras, a number of stereoscopic pictures are obtained during testing. The current 3D position of any point within the measurement field can then be determined by post processing the pictures using the software being a part of the measurement system. A spray-painted, randomly distributed black and white pattern on the surface of the test object, deforming along with the test object itself during loading, enables the in-plane strains to be assessed. Post-processing of the pictures starts with identification of a reference state, here being that of the unloaded specimen. Each picture is then subdivided into partially overlapping sections, or facets, the size and amount of overlap being set by the user on the basis of the spatial resolution and accuracy desired. The gray-scale of the pictures is utilized in performing cross-correlation calculations, such that each facet position can be tracked with subpixel accuracy from one pair of pictures to the next. This allows different strain measures pertaining from the displacement field to be calculated.

The frame-grabbing intervals of the two cameras were adjusted so that $50-100$ pictures per camera were obtained during each test. The sampling of the
Fig. 3 Overview of the test setup and the data collection system

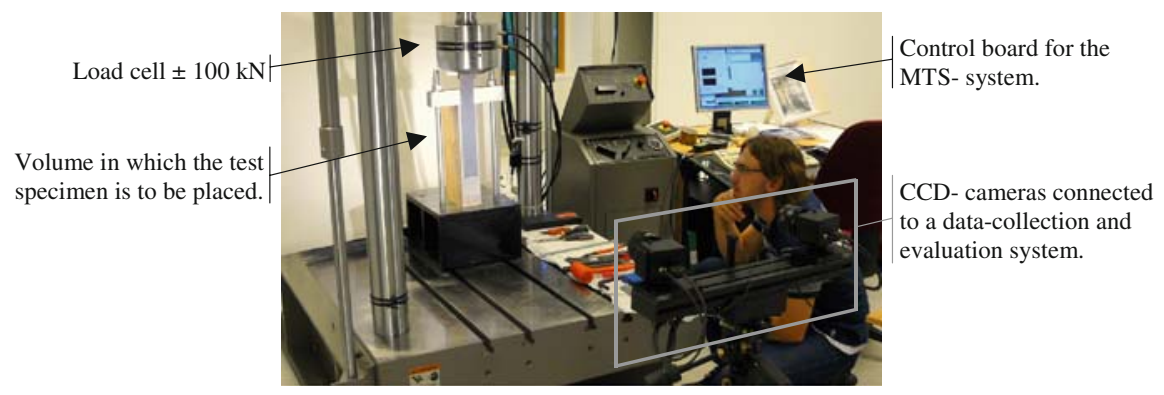


load and displacement signals from the testing machine was synchronized in time with the pictures taken.

\subsection{Analytical analysis}

Analytical models for interaction between wood and FRP involving different boundary conditions can be formulated. A detailed account of how this can be done by use of the generalized Volkersen theory, as it is called, is given by Serrano and Gustafsson [7]. As shown by e.g. Kirlin [9], the state of stress in the overlap joint can be mulitaxial. For example stresses perpendicular to the loading direction (peel direction) could develop close to the end of the FRP strip. Such stresses can, in general, be assumed to influence the level at which failure is initiated, but also the ultimate load level. The generalized Volkersen theory used here includes only longitudinal shear, although also peel stress could be included in the analysis, for example by including the shear deformation of the wood, see Gustafsson and Serrano [10]. In order to keep the analytical expression as simple as possible, the 1D-theory is used here, however.

Definitions of the symbols used in defining the specimen and the boundary conditions in question are shown in Fig. 4.

In the conventional Volkersen theory, [8], the differential equation for the shear stress present in a lap joint can be expressed as

$\tau^{\prime \prime}-\omega^{2} \tau=0$

where

$\omega^{2}=\frac{G b}{t}\left(\frac{1}{E_{2} A_{2}}+\frac{1}{E_{1} A_{1}}\right)$

In the expressions above, $G$ is the shear stiffness of the adhesive layer, $t$ represents the thickness of the adhesive layer, $b$ is the width of the adhesive layer, $E$ is the modulus of elasticity, and $A$ is the equivalent area in the wood and in the FRPs respectively. The general solution to the differential equation (1) is

$\tau(y)=C_{1} \cosh (\omega y)+C_{2} \sinh (\omega y)+\tau_{p}$

The constants $C_{1}$ and $C_{2}$ can be calculated using the boundary conditions, which for the present case are $N_{1}(0)=P, N_{2}(0)=-P, N_{1}(L)=0$ and $N_{2}(L)=0$. These conditions enable an expression of shear stress, $\tau(y)$, to be obtained:

$\tau(y)=\frac{P \omega}{b}\left(\frac{\cosh (\omega y)}{\tanh (\omega L)}-\sinh (\omega y)\right)$

This expression can be used to obtain the force $N_{1}$ by integration of the shear stress times the width $b$.

$N_{1}(y)=P\left(\cosh (\omega y)-\frac{\sinh (\omega y)}{\tanh (\omega L)}\right)$

The corresponding strains in the FRP are found to be

$\varepsilon_{1}(y)=\frac{N_{1}(y)}{E_{1} A_{1}}=\frac{P}{E_{1} A_{1}}\left(\cosh (\omega y)-\frac{\sinh (\omega y)}{\tanh (\omega L)}\right)$

This expression implies that, for any given values of the load $P$ and of the anchorage length $L$, the strains in the FRP, $\varepsilon_{1}$, within the interval $0 \leq y \leq L$ can be obtained.

The expressions above are for linear elastic materials. The so-called generalized Volkersen theory, [7], enables the fracture energy of the bond-line to be introduced as an additional parameter. This is done by introducing the equivalent shear stiffness of the adhesive layer. Accordingly, $\tau_{\mathrm{f}}^{2} /\left(2 G_{\mathrm{f}}\right)$ rather than $G / t$ is used to determine the shear stiffness, where $G_{\mathrm{f}}$ is the fracture energy of the bond line and $\tau_{\mathrm{f}}$ is the intrinsic shear strength of the bond line. Introducing the equivalent shear stiffness into (1)-(4) and assuming that the joint fails when the maximum

Fig. 4 Definitions of parameters used in the analytical equations

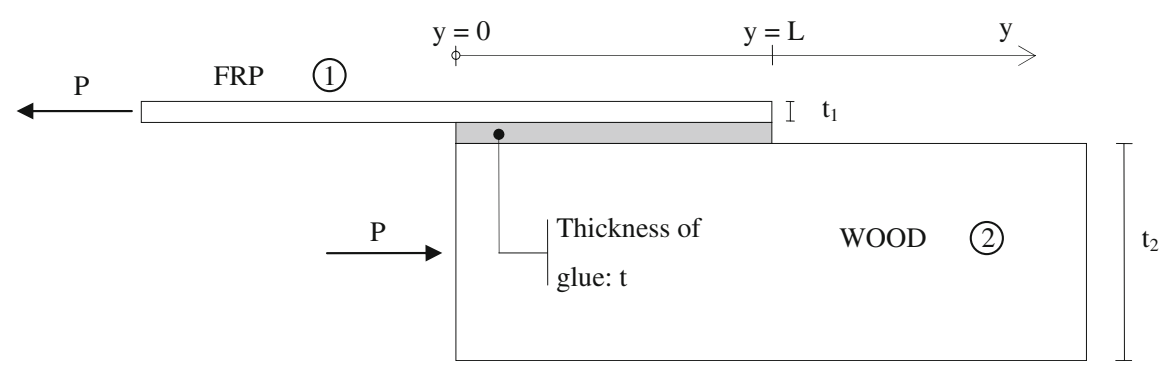


stress at the most stressed point in the bond line equals $\tau_{\mathrm{f}}$ allows an expression for the normalized strength of the lap joint to be derived:

$\frac{P_{\mathrm{f}}}{A \tau_{\mathrm{f}}}=\frac{\tanh (\omega l)}{\omega l}$

where $A$ is the area of the overlap (i.e. $A=b \times l_{\text {glue }}$ ).

For ductile joints, i.e. those in which $\omega$ approaches zero, the normalized strength equals unity. This means, in turn, that for such joints the shear stress distribution is uniform. Consequently, the governing material parameter for prediction of the load bearing capacity is the local strength of the bond line, $\tau_{\mathrm{f}}$. When $\omega$ is sufficiently large (approaching infinity), the corresponding "strength" parameter governing the load-bearing capacity of the joint is the fracture energy of the bond line. Under such conditions, the prediction of (7) yields the same result as that provided by classical linear-elastic fracture mechanics theory (LEFM), and the load bearing capacity is e.g. independent of the glued length $l$, but depends on the ratio of the adherend axial stiffnesses, $E_{1} A_{1} /$ $\left(E_{2} A_{2}\right)$. In cases between the two extreme cases above, both the local strength and the fracture energy influence the load-bearing capacity of the joint. Thus, the generalized Volkersen theory can be said to be a unifying theory.

\subsection{Analysis using the finite element method}

The test specimens were also analyzed numerically using the finite element method. Similar analyses have been carried out by Serrano [11], for example, with use of nonlinear FEM. A numerical and experimental study of glued-in rods in which strain distributions similar to those achieved in the present study has been carried out by del Senno et al. [12].

In order to perform the present analysis six material parameters, two for each material, were introduced under the assumption of the material being linearelastic isotropic, see Table 1 . This is obviously an approximation since wood, for example, is normally considered to be a strongly orthotropic material, yet for the analysis here the approximation is quite adequate, since the load is introduced primarily in the longitudinal direction of the wood. The accuracy of the FE-model was verified by use of models involving orthotropic parameters. The properties that are given for the glue should be considered as equivalent values
Table 1 Material parameters used in the finite element analyses

\begin{tabular}{lll}
\hline Material & MOE & Poissons ratio \\
\hline Wood & $10 \mathrm{GPa}$ & 0.3 \\
Epoxy, Resin 220 & $62 \mathrm{MPa}^{\mathrm{a}}$ & 0.2 \\
FRP & $150 \mathrm{GPa}$ & 0.1 \\
\hline
\end{tabular}

${ }^{\text {a }}$ Pertains to the calculated equivalent modulus of elasticity

since they include possible interface effects in the layers between the glue and the wood and between the glue and the FRP. In order to keep the model as small as possible with respect to the number of degrees of freedom, account is taken of the symmetry of the test set-up. The finite element model was loaded by displacement-controlled movement, the same way that it was done in the experiment. The boundary conditions used in the finite element model are shown in Fig. 5. Since the main purpose of the current FE-analyses was to visualize the general stress distribution along the FRP, and not to estimate a single point maximum stress value at e.g. the corners of the FRP, no mesh refinement was used in the FE-models.

\section{Results and discussion}

\subsection{Strength}

The load-bearing capacities obtained from the experiments of each of the fifteen specimens that were tested, together with the average value obtained for each of the three groups, are shown in Table 2. The average value for groups $\mathrm{A}, \mathrm{B}$ and $\mathrm{C}$ i.e. for anchorage lengths 50, 150 and $250 \mathrm{~mm}$, were 18.4, 32.8 and $35.5 \mathrm{kN}$, respectively. This shows clearly that the load-bearing capacity depends on the anchorage length, although in a non-linear fashion, and that the influence of the anchorage length diminishes as the anchorage length increases. The highest loadbearing capacity was that obtained for group $\mathrm{C}$, which had the greatest anchorage length, however in group $\mathrm{B}$, having an anchorage length $40 \%$ less, the strength is only $7.6 \%$ lower. In addition, in group A the anchorage length is $20 \%$ that of the anchorage length in group $\mathrm{C}$, whereas the load-bearing capacity for that group is nearly $50 \%$ of that obtained for group C. In Fig. 6 the average strength is shown as a function 
Fig. 5 Boundary conditions and loading of the finite element model used for simulation of the lap-joint

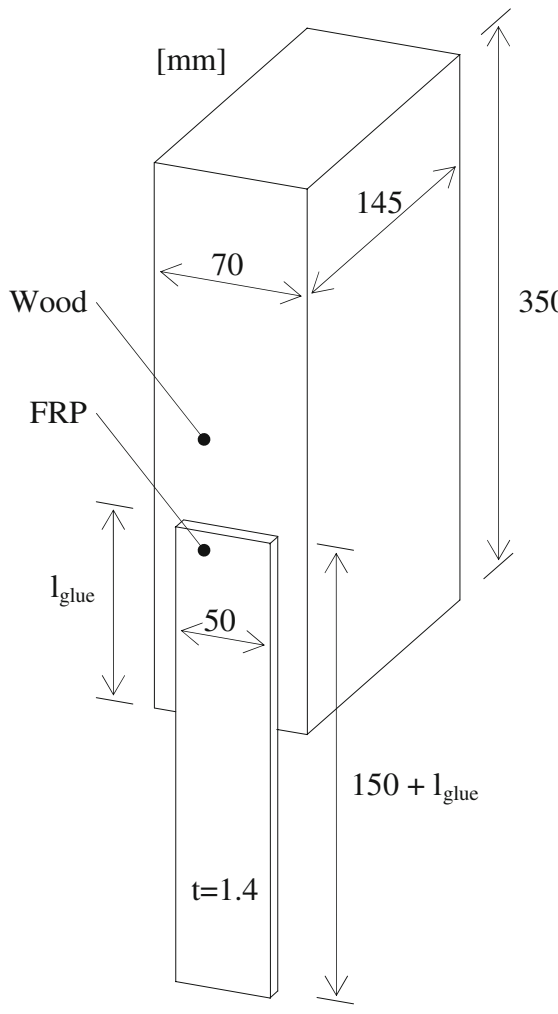

B.C. used in the FE- analysis.

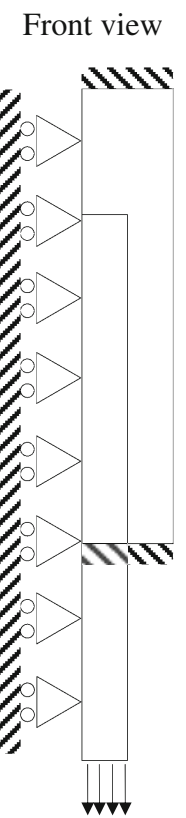

Side view

Displacement controlled

loading.
Table 2 Load-bearing capacities obtained for specimens within groups $\mathrm{A}, \mathrm{B}$ and $\mathrm{C}$ and the mean value for each group

\begin{tabular}{llll}
\hline $\begin{array}{l}\text { Group } \\
\text { Anchorage length }\end{array}$ & $\begin{array}{l}\text { A } \\
50 \mathrm{~mm}\end{array}$ & $\begin{array}{l}\text { B } \\
150 \mathrm{~mm}\end{array}$ & $\begin{array}{l}\mathrm{C} \\
250 \mathrm{~mm}\end{array}$ \\
\hline Test specimen & & & \\
1 & 16.9 & 31.5 & 33.7 \\
2 & 20.3 & 36.9 & 36.6 \\
3 & 16.4 & 31.9 & 36.5 \\
4 & 18.8 & 32.4 & 35.5 \\
5 & 19.4 & 31.1 & 35.4 \\
Average load-bearing capacity & 18.4 & 32.8 & 35.5 \\
\hline
\end{tabular}

The values are given in $\mathrm{kN}$

of the anchorage length, highlighting the highly non-linear nature of this relation.

Two different failure modes were found to occur in the experiment, one of these being shear failure of the wood material and the other a combination of failure of the wood and of the adhesive at the interface between the FRP and glue. An example of each of these two modes is shown in Fig. 7. Most common for all three different anchorage lengths was the shear failure of the wood material.

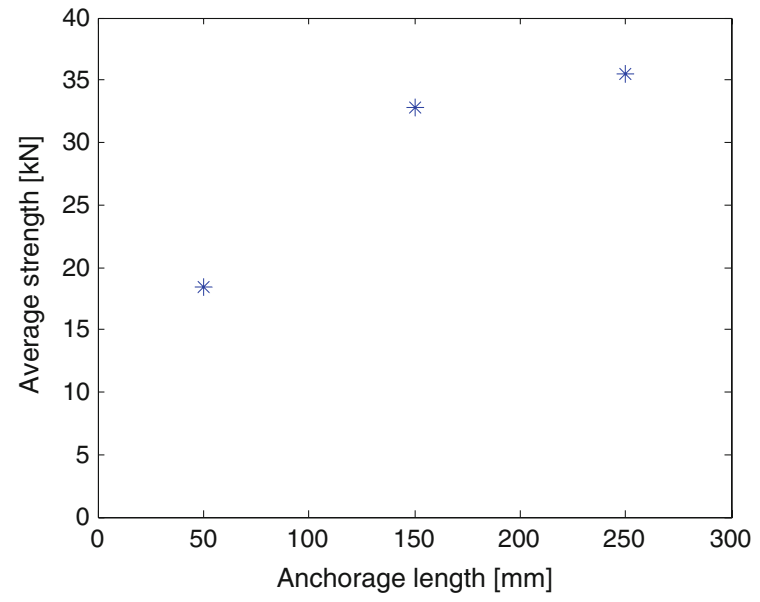

Fig. 6 Average of experimentally obtained results for anchorage lengths 50,150 and $250 \mathrm{~mm}$ respectively

The normalized strength of a joint as a function of the anchorage length is shown in Fig. 8. The analytical solution has been fitted to the experimentally obtained result in a least square sense, by varying the shear strength $\tau_{\mathrm{f}}$ and the fracture energy $G_{\mathrm{f}}$. The fit to the analytical solution yielded a value of $8.2 \mathrm{MPa}$ for the shear strength and $1700 \mathrm{Nm} / \mathrm{m}^{2}$ for the fracture 
Fig. 7 An example of each of the two failure modes that appeared: a shear failure of the wood material (test specimen A3), b combination of shear failure of the wood and adhesive failure at the junction of wood fibre and epoxy (test specimen C3)

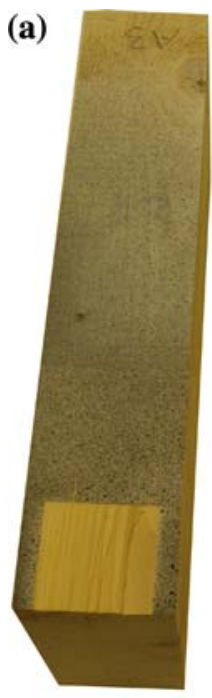

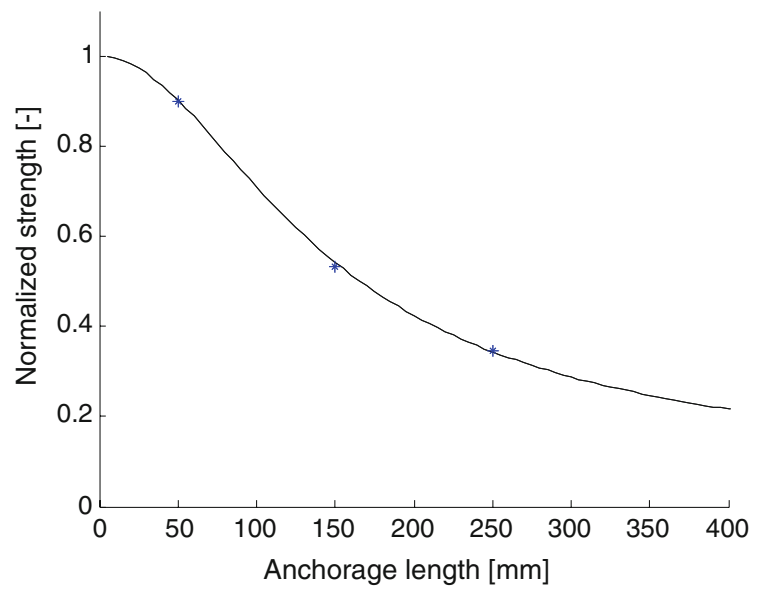

Fig. 8 Normalized strength of the joint as a function of the anchorage length. The analytical expression (8) is shown as a solid line and the measured normalized strength for each of the three groups A, B and C is marked with an asterisk

energy. The effective area in the wood was assumed to be $50 \mathrm{~mm}$ wide and $50 \mathrm{~mm}$ deep, as based on the width of the FRP and the depth of the top beam. The curve shows a given change in anchorage length to have a stronger influence on the normalized strength of the
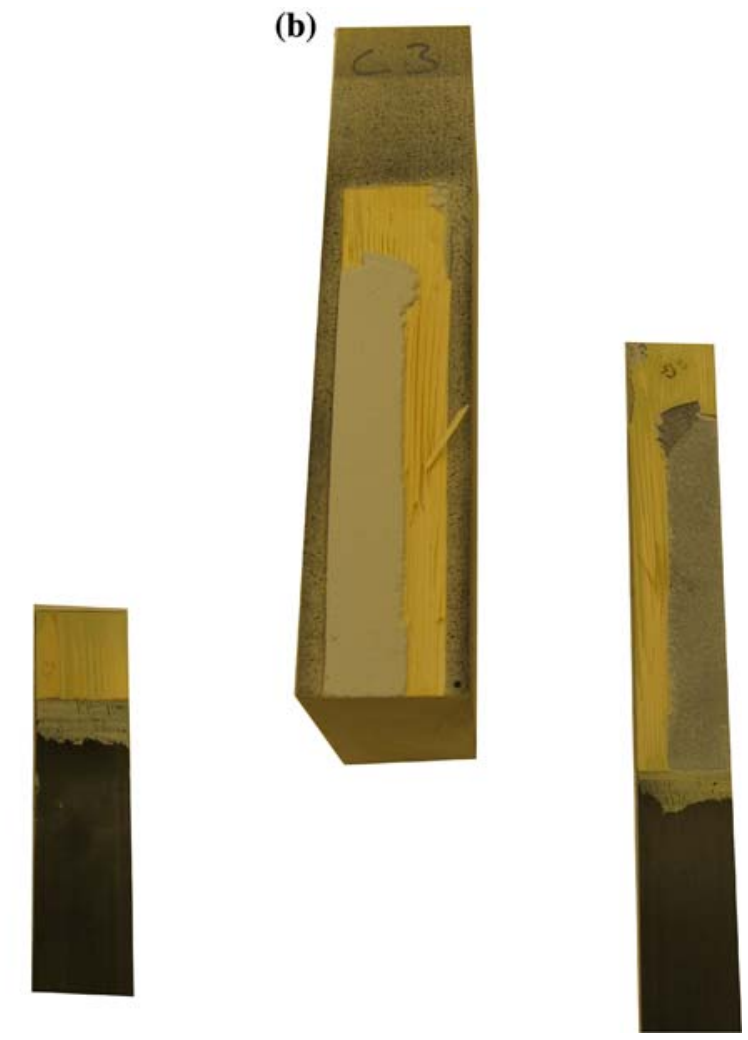

joint for shorter than for longer anchorage lengths. The shape of the curve follows closely the three mean results of the experiments. Note that more experiments should be performed in order to verify the curve for longer anchorage lengths, those longer than around $300 \mathrm{~mm}$. The results agree well with results previously obtained by e.g. Johansson et al. [2] and Steiger et al. [5].

\subsection{Stiffness}

Figure 9 shows the load-displacement response obtained in the tests. Curves based on displacement as measured by the vertical displacement of the piston and as well as curves based on displacement as measured by the contact-free system are shown. Using the curves based on the vertical displacement of the piston, the stiffness can be calculated to be approximately $30 \mathrm{MN} / \mathrm{m}$. This value is independent of the anchorage length. The differences between the plotted curves can be partly explained in terms of local phenomena in the test setup. In the curves based on the vertical movement of the piston those effects 
Fig. 9 Load-displacement curves for all 15 specimens when the vertical displacement is measured in the piston and for eight of the specimens when the vertical displacement is obtained from the contactfree (c-f) system

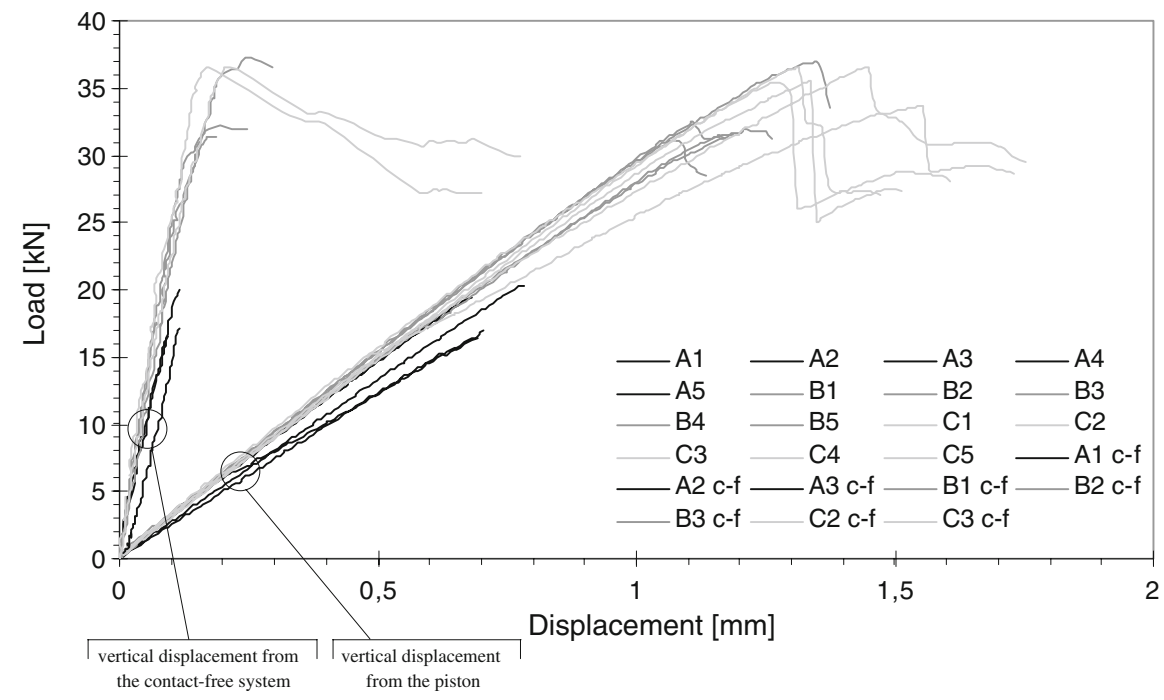

will be included in contradiction to the curves based on the contact-free system.

In order to avoid inclusion of the flexibility of the test set-up in measuring the lap-joint stiffness, test specimen numbers 1,2 and 3 in each group were evaluated with respect to the relative vertical displacement between a point P1 located on the piece of wood $10 \mathrm{~mm}$ from the upper edge and a point P2 located in the center of the FRP on the edge of the wood piece, see Fig. 10. This was easily done by use of the optical evaluation system. The relation between the load and this relative displacement, together with the load and vertical displacements as obtained from the piston, are shown in Fig. 9. For the case in which the distance between the two points is employed, the stiffness obtained is about $180 \mathrm{kN} / \mathrm{m}$, some six times as high as when the vertical position of the piston is the basis for the calculations. This difference highlights the power of using an optical system with the possibilities it provides of selecting evaluation points after testing has been performed. The accuracy of testing can be markedly increased in this way.

\subsection{Strain distribution}

It can be shown from results obtained using the contact-free system how the strain $\left(\varepsilon_{y}\right)$ varies over a given specimen. Figure 11 presents an example from each of the three groups of how strain is distributed in a test specimen. The upper part of the figure in each

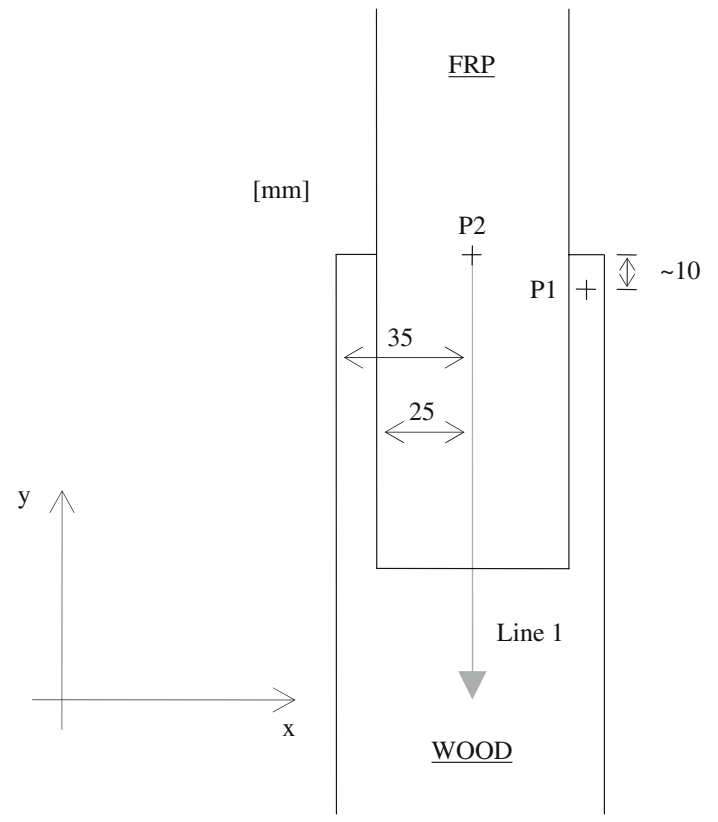

Fig. 10 Definitions of points P1 and P2 between which vertical displacements were measured, and Line 1, along which the strain component $\varepsilon_{y}$ was measured

of the three cases shows the results obtained for a given load from the contact-free system, the bottom part of the respective figure showing the results of the finite element analysis for the same load. The red colour indicates large strains and the blue colour small to negative strains. It can be readily seen that the strains in the FRP are largest close to the edge of the piece of wood, their decreasing then toward in the 

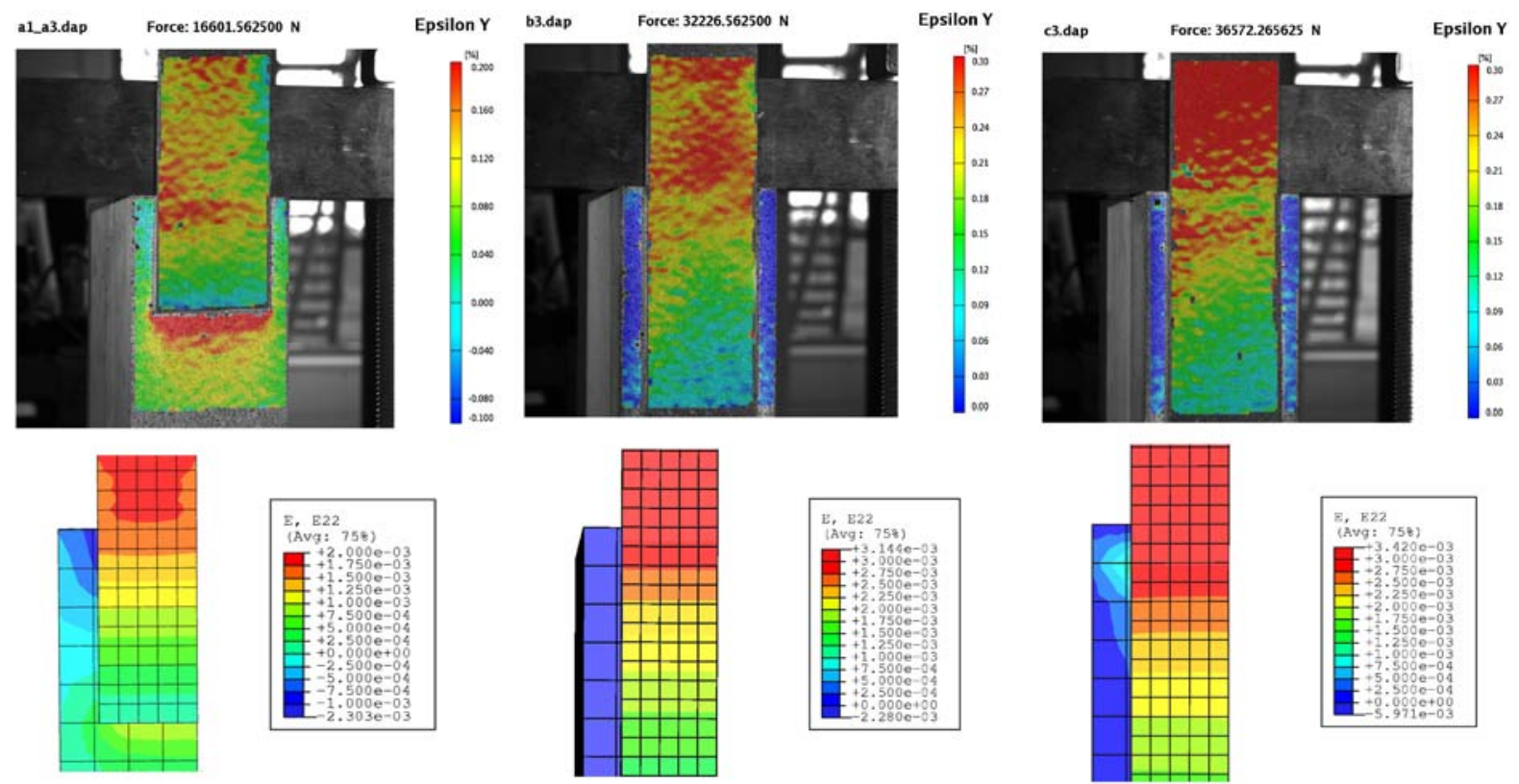

Fig. 11 Examples of strains $\varepsilon_{y}$ close to failure obtained in one of the testings in each of the three groups. $\varepsilon_{y}$ for a test specimen from group A, $F=16.6 \mathrm{kN} ; \varepsilon_{y}$ for a test specimen from group $\mathrm{B}, F=32.2 \mathrm{kN} ; \varepsilon_{y}$ for a test specimen from group $\mathrm{C}, F=36.6 \mathrm{kN}$

inner parts of the glued area. For group A it can also be seen that the strains are largest in the part of the wood toward the end of the FRP.

In Fig. 12 the normal strains in the FRP along line 1, defined as in Fig. 10, obtained in three different ways are compared with each other for group A, B and $\mathrm{C}$ respectively. The strains shown are those obtained by use of the contact-free measurement system, as well as on the basis of the analytical expression and the numerical FE-analysis. The strains obtained in the experiments were averaged within each group before being plotted in the figure. The maximum strain just prior to failure was $1.45 \%$ for group A, $2.80 \%$ for group B and $2.81 \%$ for group C.

The figure shows that the normal strains (in the FRP) at the edge are appreciably lower in group A than in groups B and C, where they are quite similar in size. The relatively linear strain distribution can be explained by the fact that the glue is of relatively low stiffness compared with the material it is glued to. A perfectly linear distribution would imply that the shear stress in the bond line is constant along the overlap. The analytical calculations, as previously indicated, are based for each of the specimens on an
Fig. 12 Moving average of the component $\varepsilon_{y}$ in the FRP at maximum load within each group. The analytical results and the results of the finite element analysis are also shown for each of the three groups

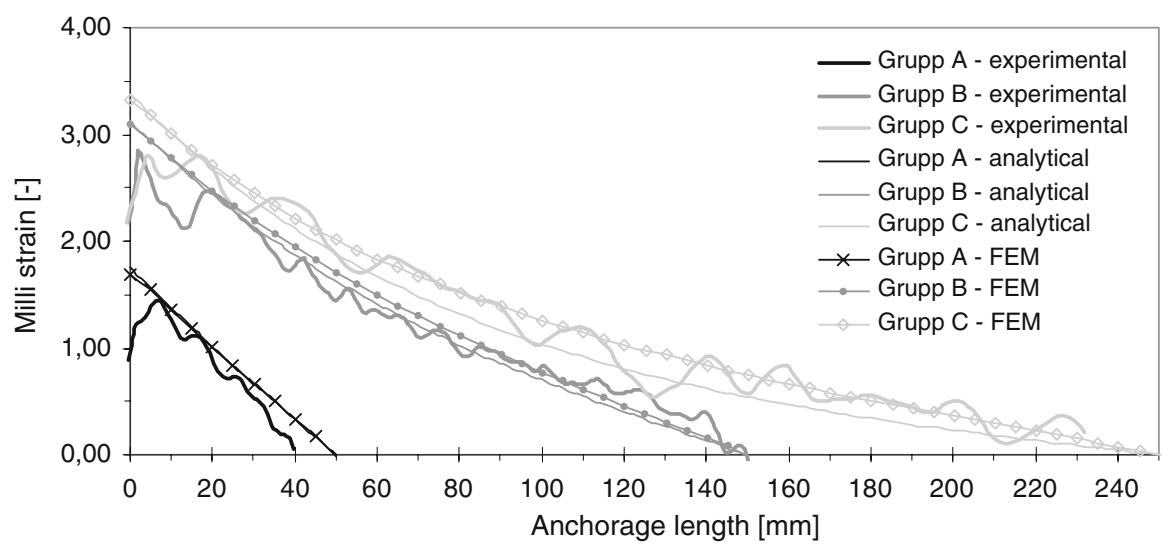


effective area of $50 \times 50 \mathrm{~mm}^{2}$ and a glue thickness of $t=1.3 \mathrm{~mm}$. The equivalent shear modulus $G$ of the glue can be calculated as having a value of 25.7 MPa according to the formula

$G=\frac{\tau_{\mathrm{f}}^{2} t}{2 G_{\mathrm{f}}}$

where the shear strength $\tau_{\mathrm{f}}=8.2 \mathrm{MPa}$ and the fracture energy $G_{\mathrm{f}}=1700 \mathrm{Nm} / \mathrm{m}^{2}$. The results of the three-dimensional FE-analysis are also shown in Fig. 12. A thickness of $t=1.3 \mathrm{~mm}$ was used for the glue, the corresponding modulus of elasticity being calculated as $62 \mathrm{MPa}$ (corresponding to a $G=$ 25.7 MPa and a Poisson's ratio of $v=0.2$ ). The agreement between the calculations and the results of the experiments is rather close, implying that calculations for the type of lap joints shown here can be done analytically using generalized Volkersen theory and numerically using linear FEM.

\section{Conclusions}

The 15 test specimens, in each of which a $50 \mathrm{~mm}$ wide and $1.4 \mathrm{~mm}$ thick laminate of reinforced fibre polymers was glued to a $70 \mathrm{~mm}$ wide piece of wood, were all loaded to failure. The specimens belonged to three different groups, those having a 50,150 and $250 \mathrm{~mm}$ long anchorage, respectively, of the FRP to the wood. The deformation and the stiffness of the joints were evaluated using an optical evaluation system. The load-bearing capacity, the stiffness and the strains were also evaluated using both analytical expressions and numerical methods. The results of the experimental investigation and of the numerical and the analytical calculations allow a number of conclusions to be drawn.

- The stiffness of the joints within the interval that was tested is basically independent of the anchorage length. For the combination of FRP, glue and wood examined the stiffness was found to be $180 \mathrm{MN} / \mathrm{m}$.

- To obtain an accurate stiffness value it is important that only the stiffness of the part that is studied be assessed. If a contact-free optical evaluation system is employed, the part to be studied can readily be chosen after the experiment has been carried out.
- The load-bearing capacity of the joint is partly a function of the anchorage length, becoming greater if the anchorage length is increased, but beyond a certain point the anchorage length appears to play only a limited role. In the experiments carried out, this was shown by the fact that, despite there being about a $40 \%$ difference in anchorage length between groups $\mathrm{B}$ and $\mathrm{C}$, the average strength of the two differed by only about $10 \%$.

- The strain was found to be at a maximum at a point close to the end of the piece of wood. The maximum strain measured prior to failure was $1.45 \%$ for group A, $2.80 \%$ for group B and $2.81 \%$ for group C (average values).

- For the type of lap-joints involved, close agreement between results of the experiments and results based on use of generalized Volkersen theory as well as of linear FE-analysis was obtained. An important aspect of the analyses carried out was to introduce the bond-line fracture energy as a major parameter.

- The load-bearing capacity of joints of the type studied depends on the local strength of the bond line, the geometry of the joint (anchorage length and cross sectional area of the adherends) the stiffness of the FRP and the wood, and on the fracture energy of the bond line.

Gluing FRPs to timber can increase both the strength and the stiffness of parts of a structure in which this is needed. In the experiments carried out, the effects of different anchorage lengths as well as of certain critical strains were examined. The close agreement obtained between the theoretical calculations and results of the tests performed show it to be possible to perform simulations in this area with a high degree of accuracy.

Acknowledgements The authors want to express their sincere gratitude to Sag $i$ Syd for the financial support to the studies.

\section{References}

1. Gustafsson P, Enquist B (1993) Advanced materials based on straw and wood Fibre reinforcement of glulam. Division of Structural Mechanics, Lund University, Lund

2. Johansson H, Blanksvärd T, Carolin A (2006) Glulam members strengthened by carbon fibre reinforcement. Mater Struct 40:47-56 
3. Kliger R, Johansson M, Crocetti R (2008) Strengthening timber with CFPR or steel plates-short and long-term performance. In: World conference on timber engineering, Miyazaki, Japan

4. Guan ZW, Rodd PD, Pope DJ (2005) Study of glulam beams pre-stressed with pultruded GRP. Comput Struct 83 : 2476-2487

5. Steiger R, Gehri E, Widmann R (2006) Pull-out strength of axially loaded steel rods bonded in glulam parallel to the grain. Mater Struct 40:69-78

6. Steiger R, Gehri E, Widmann R (2004) Glued-in steel rods: a design approach for axially loaded single rods set parallel to the grain. In: CIB-W18 meeting thirty-seven, Edinburgh, UK

7. Serrano E, Gustafsson P (2006) Fracture mechanics in timber engineering — strength analyses of components and joints. Mater Struct 40:87-96

8. Volkersen O (1938) Die Nietkraftverteilung in zugbeanspruchten Nietverbindungen mit konstanten Laschenquerschnitten. Luftfahrtforschung 15:41-47
9. Kirlin CP (1996) Experimental and finite-element analysis of stress distribution near the end of reinforcement in partially reinforced glulam. MS thesis, Department of Wood Science \& Engineering, Oregon State University, Corvallis

10. Gustafsson PJ, Serrano E (2002) Glued-in rods for timber structures-development of a calculation model. Report TVSM-3056. Division of Structural Mechanics, Lund University, Lund

11. Serrano E (2000) Adhesive joints in timber engineeringmodelling and testing of fracture properties. Report TVSM 1012. PhD-thesis, Department of Mechanics and Materials, Lund University, Lund

12. del Senno M, Piazza M, Tomasi R (2004) Axial glued-in steel timber joints-experimental and numerical analysis. Holz als Roh- und Werkstoff 62:137-146 